\title{
Cystic extraskeletal osteosarcoma: Three case reports and review of the literature
}

\author{
YOSHIHIRO ARAKI ${ }^{1}$, NORIO YAMAMOTO ${ }^{1}$, KATSUHIRO HAYASHI $^{1}$, AKIHIKO TAKEUCHI $^{1}$, \\ SHINJI MIWA ${ }^{1}$, KENTARO IGARASHI ${ }^{1}$, YUTA TANIGUCHI ${ }^{1}$, HIROTAKA YONEZAWA ${ }^{1}$, \\ SEI MORINAGA ${ }^{1}$, TAKAYUKI NOJIMA ${ }^{1,2}$ and HIROYUKI TSUCHIYA ${ }^{1}$ \\ ${ }^{1}$ Department of Orthopedic Surgery, Graduate School of Medical Sciences; ${ }^{2}$ Department of Pathology, \\ Kanazawa University, Kanazawa, Ishikawa 920-8641, Japan
}

Received November 14, 2019; Accepted January 31, 2020

DOI: $10.3892 / \mathrm{mco} .2020 .2015$

\begin{abstract}
Extraskeletal osteosarcoma is a rare soft tissue sarcoma and typically appears as a solid mass with variable mineralization. A few cases of extraskeletal osteosarcoma have shown extensive hemorrhagic changes or bleeding due to its high-grade malignancy; however, to the best of our knowledge, no previous reports have described the pathological characteristics of tumors with non-hemorrhagic cystic change. The present report discusses three cases involving cystic lesions with a solid area at the periphery that arose in soft tissues. The large cystic spaces contained only yellowish-brown fluid and little or no blood, with no clear pathological necrotic tissue. The solid component comprised a focal area of osteoid production by highly anaplastic sarcomatous cells. All of the cases showed high-grade malignancy histologically, and the average maximum diameter was approximately $175 \mathrm{~mm}$. The tumors were located at the adductor muscles in two cases and at the intermuscular region of the hamstring muscles in one case. All of the patients consulted a doctor after more than half a year had passed since the recognition of swelling or a mass on their affected extremities. Surgical treatment was performed for all patients. One patient had lymph node metastasis, and another had lymphoedema after surgery, but no histological invasion to the lymphatic ducts was observed in the excised specimen. Degenerative changes in the fluid content over a long time-course as a result of tumor bleeding or necrosis, rather than lymphorrhea by lymphatic channel invasion of the tumor, might have contributed to the formation of the cystic lesion. Only 1 patient who underwent chemotherapy remained clinically disease-free over 10 years after surgery. Chemotherapy regimens for osteosarcoma rather than those for soft tissue sarcoma are mostly effective for extraskeletal osteosarcoma, so
\end{abstract}

Correspondence to: Professor Norio Yamamoto, Department of Orthopedic Surgery, Graduate School of Medical Sciences, Kanazawa University, 13-1 Takaramachi, Kanazawa, Ishikawa 920-8641, Japan

E-mail: norinori@med.kanazawa-u.ac.jp

Key words: extraskeletal osteosarcoma, cystic change, non-hemorrhagic fluid, no necrotic tissue, long time clinical course the diagnosis by a biopsy is essential. It is important to consider extraskeletal osteosarcoma in the differential diagnosis of soft tissue tumor with cystic form and calcification and a long clinical course before consulting a doctor.

\section{Introduction}

Extraskeletal osteosarcoma is a rare sarcoma of the non-skeletal tissue that produces neoplastic osteoid or bone (1). The tumor typically appears as a solid mass with variable mineralization and seldom has a cystic lesion.

Roller et al, reported the features of 19 patients with extraskeletal osteosarcoma radiographically and pathologically (2). In their report, mineralization on computed tomogram (CT) was seen in only $26 \%$ (5 of 19) of cases, and central necrosis on magnetic resonance imaging (MRI) was observed in $47 \%$ (9 of 16) of cases. They confirmed osteoid formation in the histology of all specimen. However, they did not mention whether or not central necrosis was observed pathologically in the excised specimen of the tumors.

Extraskeletal osteosarcoma is classified into six subtypes, just like intraosseous osteosarcoma, in the 2013 WHO classification (1). Osteosarcoma with a cystic lesion can be classified as the telangiectatic-type, although this type typically manifests as tumors predominantly composed of cystic spaces filled with blood (3). Telangiectatic-type intraosseous osteosarcoma is occasionally seen, and telangiectatic-type extraskeletal osteosarcoma that meets the strict diagnostic criteria is rarely reported (4-6). However, no previous reports have described the gross and pathological characteristics of extraskeletal osteosarcoma with non-hemorrhagic cystic change.

When a soft tissue tumor consisting of a solid lesion with calcification and a cystic lesion are observed, both benign (myositis ossificans and hemangioma) and malignant entities (dedifferentiated liposarcoma, synovial sarcoma, malignant peripheral nerve sheath tumor and undifferentiated pleomorphic sarcoma) are included in the differential diagnosis (2). It is very important to make an accurate pathological diagnosis in order to facilitate appropriate treatment with a chemotherapy regimen suited for the histological type. The clinical course and imaging findings are also useful for making a diagnosis, especially in cases of rare tumors. 
We herein report three rare cases of extraskeletal osteosarcoma with cystic change. The Ethical Institutional Review Board of Kanazawa University Hospital approved the present study, and written informed consent was obtained from all patients.

\section{Case report}

Case 1. The patient was an 81-year-old man who presented with a 2-year history of a gradually enlarging mass in his left thigh. A large, ill-defined, non-movable, non-tender, firm mass was noted on the upper medial portion of the left thigh. Plain film showed a large soft tissue mass with numerous small calcifications, located more or less centrally (Fig. 1A). CT showed a large deep-seated mass in the adductor magnus muscle (Fig. 1B). On MRI, the cystic lesion appeared hypointense on T1-weighted images and hyperintense on T2-fat suppression images, and the solid lesion showed a heterogeneous intensity on T2-fat suppression images (Fig. 1D). An axial view of dynamic MRI showed enhancement of the tumor periphery and the solid lesion (Fig. 1C). Scintigraphy with thallium-201 showed the increased uptake of the whole tumor, which was more pronounced in the solid component than in the cystic lesion. No distant metastases were observed on a close examination of the whole body.

A needle biopsy revealed undifferentiated pleomorphic sarcoma. We considered the possibility of extraskeletal osteosarcoma, but a biopsy showed no sign of malignant osteoid formation.

Surgical treatment was selected because the patient was already of advanced age and might not tolerate chemotherapy. The tumor was widely excised with the adductor magnus, semimembranosus and semitendinosus, preserving the femoral vessels, nerve and sciatic nerve. The surgical margins were negative for tumor involvement. The patient received no other adjuvant treatment. About three months after surgery, lower leg lymphoedema appeared. He was observed conservatively at an outpatient clinic but did not improve significantly. At one and half a year after surgery, recurrence was detected on imaging and confirmed by a needle biopsy. He underwent reoperation and adjuvant chemotherapy with the Adriamycin regimen. However, he was dead from his disease at three years after the primary surgery.

Case 2. A 78-year-old man presented with a soft tissue mass on the left upper posterior thigh. The mass in the adductor muscles gradually grew for a few years and was ill-defined, painless, firm, and non-movable. Radiologic findings showed both a solid and cystic lesion; the solid component included calcification on X-ray and CT, and the cystic component showed fluid-fluid levels on MRI (Fig. 2A, B and C). No distant metastases were observed on a close examination of the whole body. The tumor was pathologically diagnosed as undifferentiated pleomorphic sarcoma by a needle biopsy.

As the patient was already of advanced age and potentially unable to tolerate chemotherapy, wide excision was planned for local control. The tumor was widely excised with the adductor magnus, semimembranosus, semitendinosus and biceps femoris, preserving the sciatic nerve. A diagnosis of extraskeletal osteosarcoma was made. No invasion to the lymphatic ducts was observed in the excised specimen. The surgical margins were negative for tumor involvement. There was no hemorrhaging in the cystic spaces of the tumor, and only a yellowish-brown fluid with little blood flowed from the tumor when the resected specimen was cut (Fig. 3B).

There was no other adjuvant treatment; however, solitary lymph node metastasis was detected on MRI five months after excision of the primary tumor. The patient received oral pazopanib after the excision of the involved lymph node. However, lung metastases developed 10 months after the first surgery. Cyclophosphamide was administered as palliative chemotherapy. The patient died approximately one year after the detection of pulmonary metastasis.

Case 3. The patient was a 33-year-old woman with a soft tissue tumor in the distal part of the left posterior thigh. The patient had noticed a mass that had been gradually growing in size for approximately six months. She felt a well-circumscribed, non-tender firm mass between the biceps femoris muscle and semitendinosus muscle. CT showed a solid component with foci of calcification (Fig. 4A). On MRI, the tumor consisted of both a solid lesion and a cystic lesion. The solid lesion appeared hypointense on T1-weighted images and hypointense to hyperintense on T2-weighted images. The cystic lesion appeared hypointense on T1-weighted images and hyperintense on T2-weighted images (Fig. 4B). The periphery of the tumor and solid lesion was highlighted on enhanced MRI (Fig. 4C). No distant metastases were observed on a close examination of the whole body. The tumor was diagnosed as extraskeletal osteosarcoma by a needle biopsy.

She received a neo-adjuvant chemotherapy regimen for osteosarcoma (adriamycin and cisplatin) followed by surgery. The tumor showed complete remission after 5 cycles of chemotherapy and was widely excised with the semimembranosus and semitendinosus, preserving the sciatic nerve. The surgical margins were negative for tumor involvement. After neoadjuvant chemotherapy had been completed, a decreased renal function was observed, so the adjuvant chemotherapy regimen was changed. She was given three cycles of adjuvant chemotherapy with ifosfamide and etoposide and discharged. She is being regularly observed at an outpatient clinic. At present, over 10 years from the time of the diagnosis, she remains alive and free of disease.

\section{Pathological examination procedures and findings}

Immunohistochemical staining. For fixation of operatively extracted specimen, 10\% formaldehyde (Muto Pure Chemicals Co., Ltd.) was used at room temperature. After immersing in the formaldehyde for $24 \mathrm{~h}$, paraffin-embedded specimen were made by Tissue-Tek VIP ${ }^{\circledR}$ 6AI (Sakura Finetek Japan Co., Ltd.). Four-micrometer-thick sections cut from the representative block of each tumor were deparaffinized. The preparations were autoclaved in citrate buffer ( $\mathrm{pH}$ 6.0), and endogenous peroxidase activity was blocked with $3 \%$ hydrogen peroxide. The following primary antibodies were used: Anti-alpha-smooth muscle actin mouse monoclonal (M0851, dilution 1:100; DAKO A/S, Glostrup), anti-desmin mouse monoclonal (M0760, dilution 1:100; DAKO A/S, Glostrup), anti-S-100 rabbit polyclonal (Z0311, dilution 1:5,000; DAKO A/S, Glostrup), anti-cytokeratin mouse monoclonal (IS053, no dilution; DAKO A/S, Glostrup), anti-epithelial membrane antigen (EMA) mouse monoclonal 
A

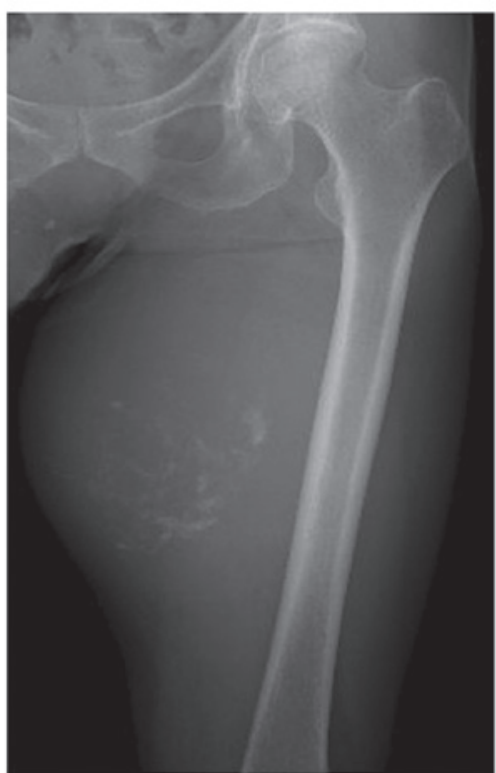

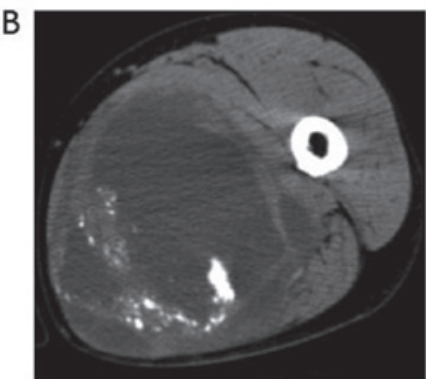

$\mathrm{C}$

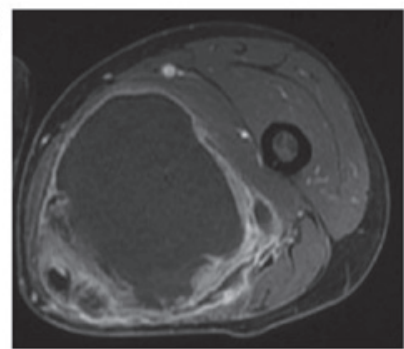

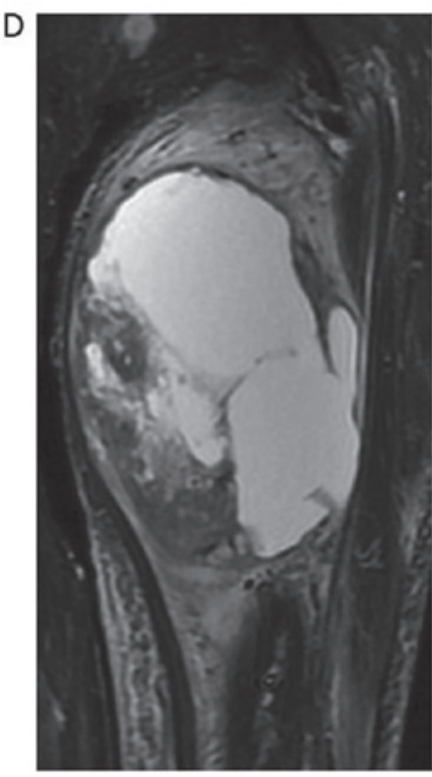

Figure 1. (A) X-ray showing a large soft tissue mass with numerous small calcifications. (B) CT shows a large deep-seated mass in the adductor magnus muscle, which is fairly circumscribed and has both a solid and cystic component. (C) Dynamic MRI shows enhancement of the capsule of the cyst and the solid component itself. (D) On T2-weighted images with fat suppression (coronal view), there is a high signal intensity in the cystic component and a low-to-isointense signal in the solid component.
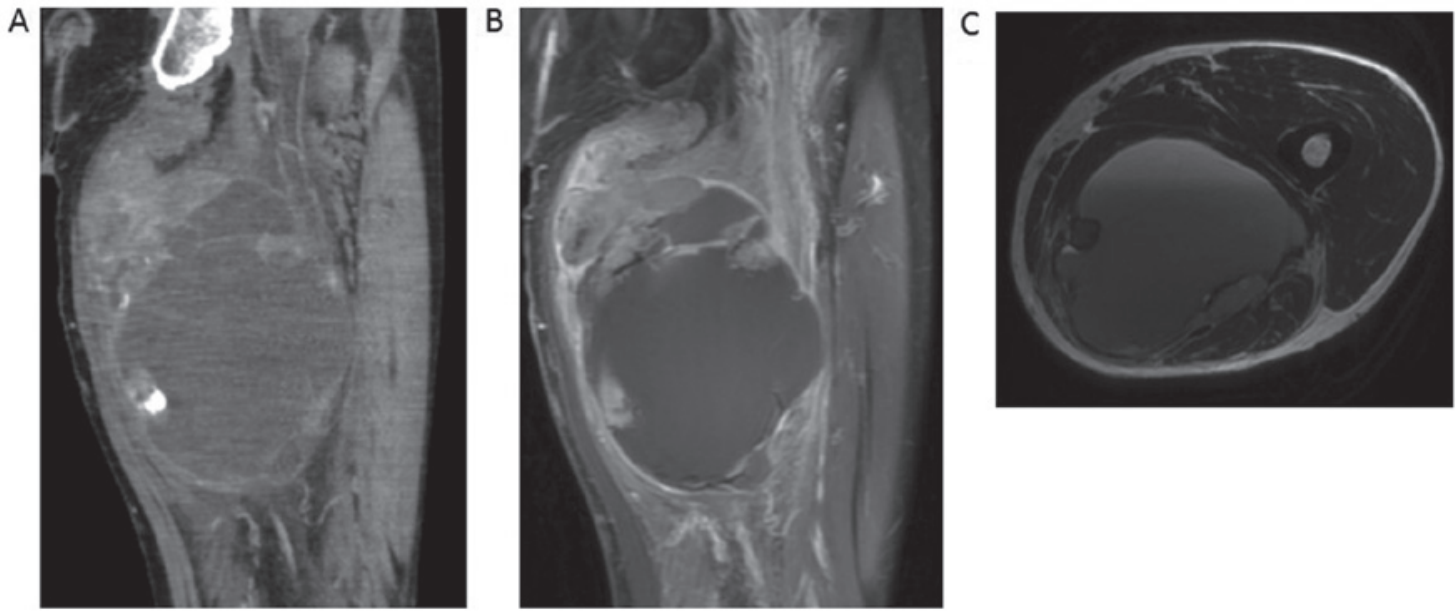

Figure 2. (A) CT shows that the tumor consists of large cystic component and a small solid component with calcification. (B) Dynamic MRI shows enhancement of the cyst periphery and the solid component. (C) Fluid-fluid levels can be seen on T2-weighted MR images (axial view).

(IS629, no dilution; DAKO A/S, Glostrup), anti-D2-40 (Podoplanin) mouse monoclonal (M3619, dilution 1: 50; DAKO A/S, Glostrup) and anti-Ki-67 rabbit monoclonal (RM-9106-S, dilution 1: 100; Thermo Fisher Scientific Anatomical Pathology). Slides were incubated for $1 \mathrm{~h}$ at room temperature with the primary antibody, and subsequently labelled by use of the secondary antibody (Histofine ${ }^{\circledR}$ Simple Stain MAX PO (MULTI), Nichirei Biosciences Inc.). The sections were examined with a confocal microscope (Olympus).

Fluorescence in situ hybridization. The probes used for the fluorescence in situ hybridization (FISH) analyses were as follows; Vysis LSI MDM2 Spectrum Orange Probe, Vysis CEP (D12Z3) (alpha-satellite) Spectrum Green Probe (Abbott Molecular Inc.), and KreatechTM CDK4 (12q13)/SE12FISHprobe (Leica Biosystems). The tissue sections were counterstained in phosphate-buffered saline containing 4',6-diamidine-2'- phenylindole dihydrochloride (DAPI II Counterstain; Abbott Molecular Inc.), p-phenylenediamine, and glycerol (Abbott Molecular Inc.), then examined with a fluorescence microscope (Olympus) equipped with a Triple Bandpass FilterTM set (Abbott Molecular Inc.) for detecting DAPI II, Spectrum Orange, and Spectrum Green.

Pathological findings. Grossly, the tumors of the three cases were fairly defined and predominantly consisted of several large cystic spaces with non-hemorrhagic fluid content (Fig. 3A) or a small amount of hemorrhaging (Fig. 3B). A grayish, tan-white, fleshy solid component was noted on the periphery of the cysts (Fig. 3A and B). Microscopically, the osteoid was located in the septa of the cyst wall (Fig. 5A). On a low-power view, the tumor was composed of haphazardly arranged, highly 
anaplastic sarcomatous cells. On a higher-power view, spindle cells with enlarged, hyperchromatic and pleomorphic nuclei were observed. There were numerous typical and atypical mitoses. Scattered tumor giant cells were also seen. Focal areas of osteoid production by sarcomatous tumor cells were seen (Fig. 5B, C and D).

Case 3 was diagnosed with extraskeletal osteosarcoma based on the histological analysis of a biopsy specimen (Fig. 5D). Neoadjuvant chemotherapy was very effective, and necrosis was observed in more than $95 \%$ of the area of the resected specimen (Fig. 5E).

Immunohistochemistry revealed focal positivity for alpha-smooth muscle actin but negativity for desmin, S100 protein, cytokeratin, and EMA. There were no lymphatic ducts with positivity for D2-40 in the area of tumor involvement. The Ki-67 proliferation index was approximately $30 \%$. The MDM2 gene was not amplified on FISH in any of the three cases (Fig. 5F). The cdk4 gene amplification was not observed on FISH in any of the three cases.

\section{Discussion}

Extraskeletal osteosarcoma is a rare soft tissue sarcoma that accounts for approximately 1-2\% of all soft tissue sarcomas and $2-5 \%$ of all osteosarcomas (1). Various etiologies of extraskeletal osteosarcoma, such as previous trauma or radiotherapy, have been reported but the actual cause of this tumor is unknown $(1,7,8)$. Approximately $10 \%$ of cases show extensive hemorrhagic change (1); however, no reports have described a tumor with a non-hemorrhagic fluid lesion, as was observed in our cases.

Roller et al, reported the radiographical and pathological features of 19 cases of extraskeletal osteosarcoma (2). They noted mineralization on CT in only $26 \%$ (5 of 19) of cases, while central necrosis on MRI was observed in 47\% (9 of 19). Regarding central necrosis, they did not clearly confirm the pathological characteristics of the specimen. Cystic lesions were observed in all of our cases on MRI, but central necrosis was not observed in the pathological examination of the specimens, in which only non-hemorrhagic fluid was found.

Cases of high-grade extraskeletal osteosarcoma are reported to be larger in size and to have more necrosis than low-grade cases (2,9-11). In all of our cases, the maximum diameter was $>17 \mathrm{~cm}$, and a histological examination revealed high-grade malignancy (Table I). On MRI, the cystic lesion findings were typically consistent with necrosis. However, there was no clear necrotic tissue. Only yellowish-brown fluid with little or no blood flowed from the tumor when the resected specimen was cut. A histological examination of the tumor did not show central necrosis. These findings suggest that the necrotic lesion or bleeding in the tumor may have gradually changed to fluid content over a long time-course. Indeed, more than half a year had passed between the patients first noticing the mass and the diagnosis, which might have been a sufficient duration to allow a change to non-hemorrhagic fluid. Case 2 showed fluid-fluid levels on MRI before surgery, but no hemorrhaging was noted in the resected tumor, and only a small amount of blood flowed with yellowish-brown fluid on cutting the specimen. This might indicate the course of changing to non-hemorrhagic fluid.

The tumors in our cases were located at the adductor muscles of the thigh in two patients and at the intermuscular region of 
A

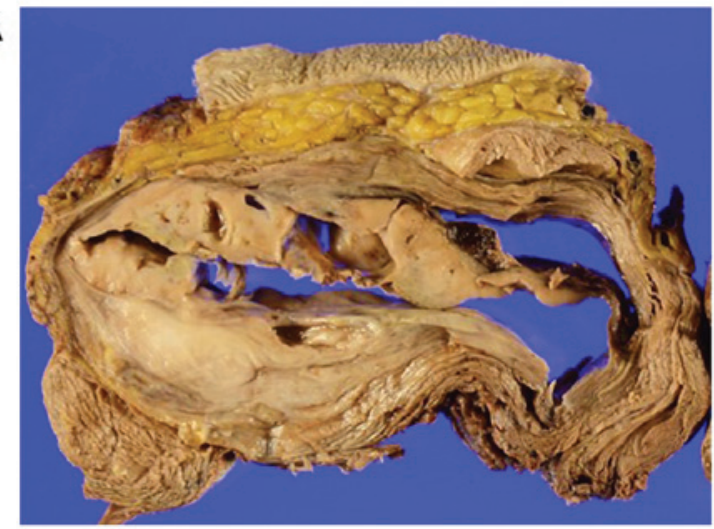

B

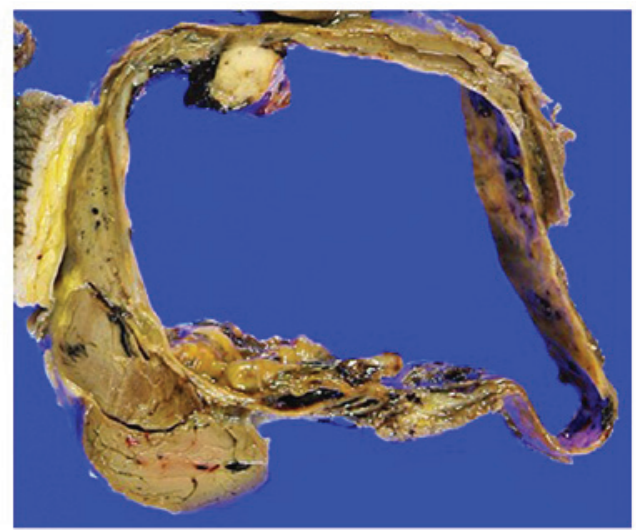

Figure 3. The tumor is fairly well defined, and a gross examination reveals it to predominantly consist of several large cystic spaces with (A) non-hemorrhagic fluid content or a small amount of (B) hemorrhaging. In Case 2, fluid-fluid levels can be seen on MR images, but only yellowish-brown fluid with little blood flowed from the tumor when the resected specimen was cut. A grayish, tan-white, fleshy solid component is noted at the periphery of the cysts (A. specimen of Case 1, B. the specimen of Case 2).

A

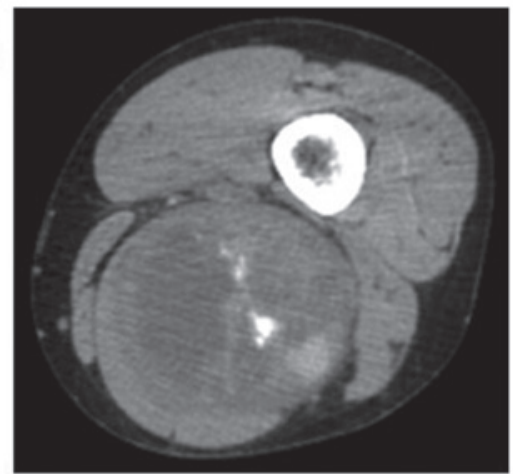

B

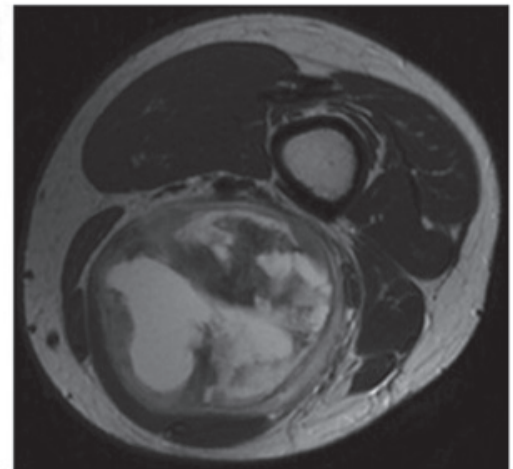

C

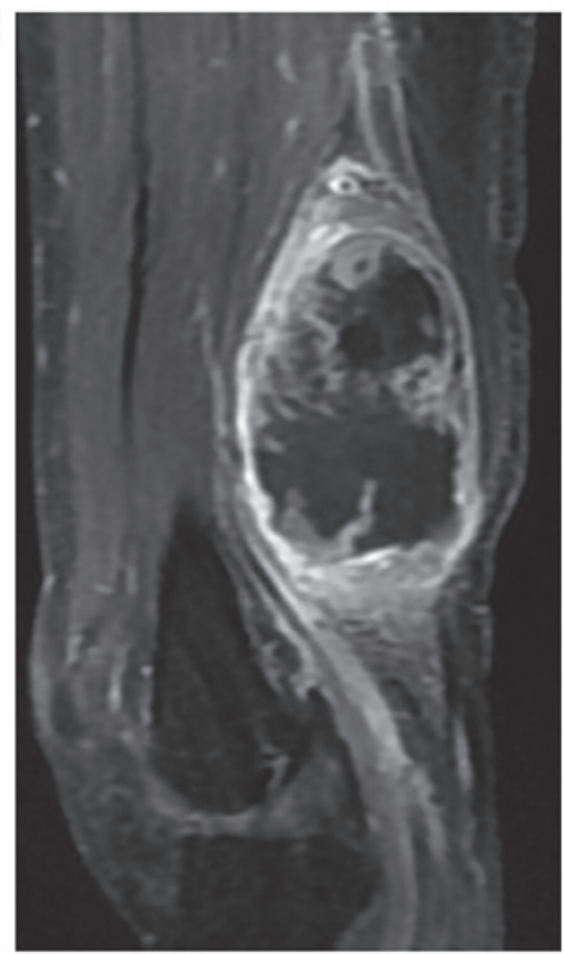

Figure 4. (A) CT shows a solid and cystic component and foci of calcification. (B) On T2-weighted MR images with fat suppression (axial view), the cystic component shows a high signal intensity, while the solid component and septa of the cyst show a low-to-isointense signal intensity. (C) Dynamic MRI shows the enhancement of the capsule and the septa of the cyst along with the solid component.

the hamstrings in one patient, where lymphatic channels from the lower legs are abundantly gathered. Lymph node involvement is likely associated with extraskeletal osteosarcoma and intratumoral lymphorrhea due to lymphatic channel invasion were assumed to have been the cause of the cystic lesion (12). However, there were no tumor-involved areas positive for D2-40, a specific marker of lymphatic ducts, in any surgical specimens.

At present, extraskeletal osteosarcoma is classified into six subtypes, just like intraosseous osteosarcoma, in the 2013 WHO classification (1). Intraosseous osteosarcoma with cystic change is typically diagnosed as telangiectatic osteosarcoma, but the cyst of intraosseous osteosarcoma is necessarily filled with blood (2). The criteria for the diagnosis of telangiectatic osteosarcoma are as follows: The absence of sclerosis on plain film imaging; a purely lytic lesion; and a predominant composition of cystic spaces filled with blood. Microscopically, the tumor comprises a sarcomatous component lining the septa and the presence inconspicuous osteoid production. In our cases, the tumors had marked cystic degeneration with little or no blood. An abundant mineralization pattern was also seen on radiography, and abundant osteoid production was pathologically observed in the specimen. With such features, the lesion did not meet the criteria for telangiectatic-type extraskeletal osteosarcoma and was appropriately diagnosed as extraskeletal osteosarcoma with cystic change. We have 
A

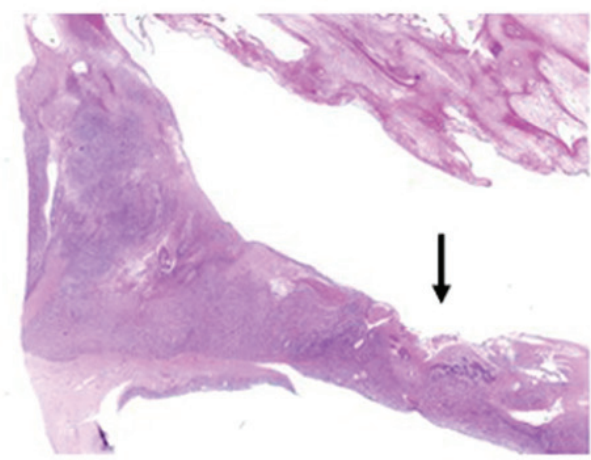

C

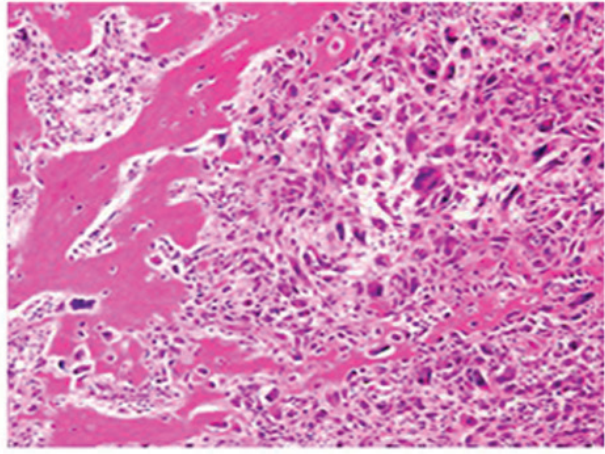

$\mathrm{E}$

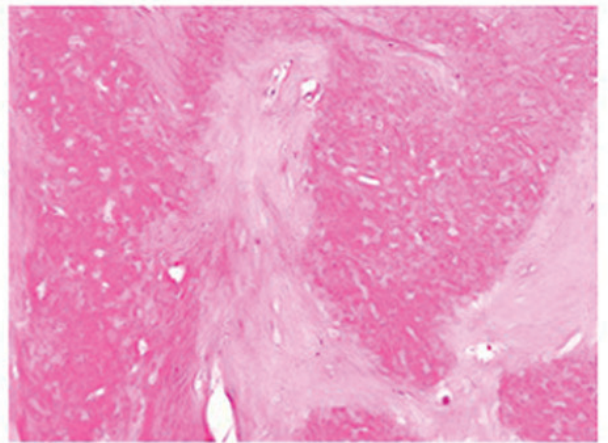

B

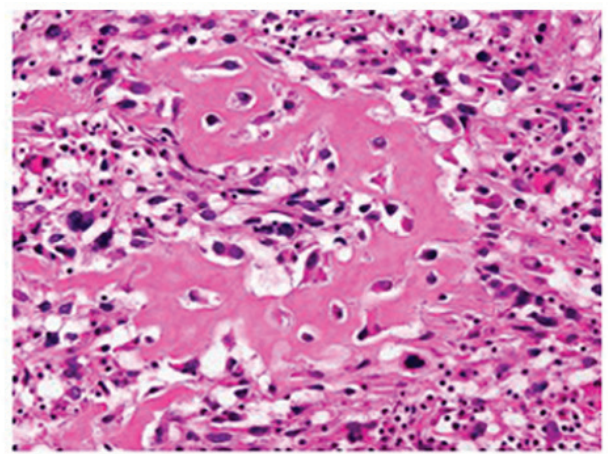

D

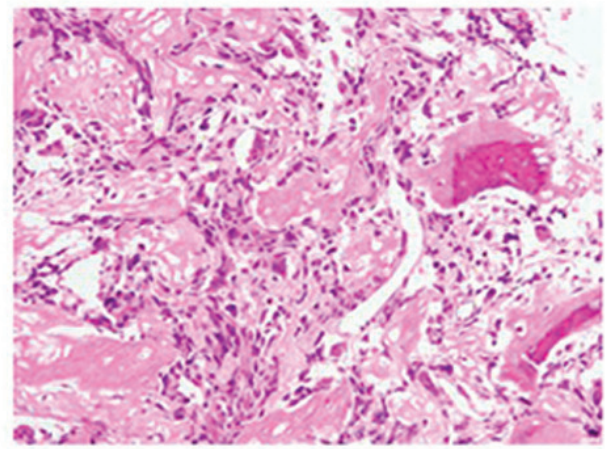

F

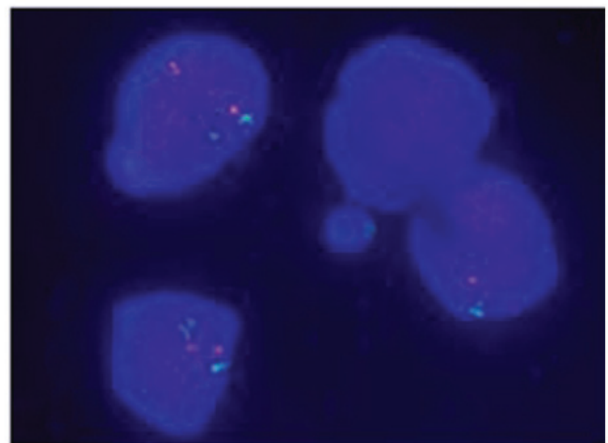

Figure 5. (A) The osteoids (black arrow) are located in the septa of the cyst wall (Case 1). Magnification, x40; hematoxylin and eosin stain. (B) The tumor consists of highly atypical spindle cells, arranged in a disorganized or vague storiform pattern, with pleomorphic nuclei and brisk mitotic activity (Case 1). Magnification, x400; hematoxylin and eosin stain. Focal areas of lace-like osteoid production by sarcomatous tumor cells are seen in the (C) wide excision specimen. (Case 2) and in the (D) needle biopsy specimen (Case 3). Magnification, x200, hematoxylin and eosin stain. (E) All tumor cells were necrotized after neoadjuvant chemotherapy with an osteosarcoma regimen (Case 3). Magnification, x200, hematoxylin and eosin stain. (F) The MDM2 gene was not amplified by fluorescence in situ hybridization (Case 1).

never encountered a case of intraosseous osteosarcoma with a non-hemorrhagic cyst. However, rare cases of cystic extraskeletal osteosarcoma do exist, as seen in the present cases. A different entity from intraosseous osteosarcoma may therefore exist among extraskeletal osteosarcoma cases.

The clinical prognosis of patients with extraskeletal osteosarcoma is poor because these patients typically show high-grade malignancy (1). Surgical resection is the standard treatment; however, perioperative chemotherapy may improve the survival (13-15). Although extraskeletal osteosarcoma is classified as a soft tissue sarcoma, chemotherapy regimens for osteosarcoma are more effective than those for soft tissue sarcoma $(16,17)$. Thus, an accurate pretreatment diagnosis of extraskeletal osteosarcoma is very important for acquiring a good survival. Biopsy specimens are essential for differentiating the entity from benign tumors or other soft tissue sarcomas. The increased vascularity or arteriovenous malformation that is often seen in children, adolescents and young adults with a hemangioma $(18,19)$; or the zonal pattern of peripheral ossification that is typically seen with myositis ossificans were not observed in our cases (20). MDM2 amplification is useful for ruling out dedifferentiated liposarcoma with osteogenic differentiation; recently, however, MDM2 amplification has been reported in extraskeletal osteosarcomas, and caution is required when MDM2 amplification is observed (21-23). Synovial sarcoma sometimes shows similar imaging findings, but the histological morphology differs from the pleomorphism of extraskeletal osteosarcoma. Malignant peripheral nerve sheath tumors are a type of neurogenic malignant tumor and sometimes show heterotopic differentiation; however, immunohistochemical staining is positive for neurogenic markers, such as S-100 protein, in most cases. Case 3 was diagnosed as extraskeletal osteosarcoma based on the histological analysis of a biopsy specimen, and neoadjuvant chemotherapy for osteosarcoma was administered. Necrosis 
was observed in $>95 \%$ of the area of the operation specimen. A good response to chemotherapy is a prognostic factor (24), and Case 3 has achieved a long survival (>10 years) with a disease-free condition.

Extraskeletal osteosarcoma can show diverse radiological findings; however, more than half a year had passed since the patients had first become aware of their tumors, which may have resulted in relatively specific imaging characteristics. It is important to consider extraskeletal osteosarcoma as a differential diagnosis of soft tissue tumors with calcification and a large cystic lesion, especially in cases with a long clinical course before consulting a doctor. Larger-scale studies will be required in order to clarify the clinical implications of this category of extraskeletal osteosarcoma.

\section{Acknowledgements}

The authors would like to thank Higuchi Takashi, Abe Kensaku, and Asano Youhei (all, Department of Orthopaedic Surgery, Graduate School of Medical Sciences, Kanazawa University, Kanazawa, Japan) for assistance in the data collection for the current study.

\section{Funding}

No funding was received.

\section{Availability of data and materials}

All data generated or analyzed during the present study are included in this published article.

\section{Authors' contributions}

HT, NY, KH and AT performed the surgery and managed the patients postoperatively. TN made a diagnosis of extraskeletal osteosarcoma with cystic change pathologically. HT, NY, $\mathrm{SMi}, \mathrm{KI}$ and TN contributed to the concept and design of the study and to the acquisition, analysis or interpretation of working data. YT, HY and SMo assisted in data collection. YA analyzed all the patient's data and was involved in drafting the manuscript. All authors read and approved the final manuscript.

\section{Ethics approval and consent to participate}

The study was approved by the Ethical Institutional Review Board of the Kanazawa University Hospital [approval no. 2019-61(3094)], and written informed consent was obtained from all study participants.

\section{Patient consent for publication}

The consent for publication of the manuscript and the related images from the patients and/or their relatives was obtained by the Kanazawa University Hospital.

\section{Competing interests}

The authors declare that they have no competing interests.

\section{References}

1. Fletcher CDM, Bridge JA, Hogendoorn P and Mertens F (eds): Extraskeletal osteosarcoma. In: WHO Classification of Tumours of Soft Tissue and Bone. 4th edition. IARC Press, Lyon, pp161-162, 2013.

2. Roller LA, Chebib I, Bredella MA and Chang CY: Clinical, radiological, and pathological features of extraskeletal osteosarcoma. Skeletal Radiol 47: 1213-1220, 2018.

3. Matsuno T, Unni KK, McLeod RA and Dahlin DC: Telangiectatic osteogenic sarcoma. Cancer 38: 2538-2547, 1976.

4. Mirra JM, Fain JS, Ward WG, Eckardt JJ, Eilber F and Rosen G: Extraskeletal telangiectatic osteosarcoma. Cancer 71: 3014-3019, 1993.

5. Dubec JJ, Munk PL, O'Connell JX, Lee MJ, Janzen D, Connell D, Masri B and Logan PM: Soft tissue osteosarcoma with telangiectatic features: MR imaging findings in two cases. Skeletal Radiol 26: 732-736, 1997.

6. Lee KH, Joo JK, Kim DY, Lee JS, Choi C and Lee JH: Mesentric extraskeletal osteosarcoma with telangiectatic features: A case report. BMC Cancer 7: 82, 2007.

7. Healy C, Kahn LB and Kenan S: Subcutaneous extraskeletal osteosarcoma of the forearm: A case report and review of the literature. Skeletal Radiol 45: 1307-1311, 2016.

8. Savant D, Kenan S, Kenan S and Kahn L: Extraskeletal osteosarcoma arising in myositis ossificans: A case report and review of the literature. Skeletal Radiol 46: 1155-1161, 2017.

9. Bane BL, Evans HL, Ro JY, Carrasco CH, Grignon DJ, Benjamin RS and Ayala AG: Extraskeletal osteosarcoma: A clinicopathologic review of 26 cases. Cancer 65: 2762-2770, 1990.

10. Thampi S, Matthay KK, Boscardin WJ, Goldsby R and DuBois SG: Clinical features and outcomes differ between skeletal and extraskeletal osteosarcoma. Sarcoma 2014: 902620, 2014.

11. Schaefer IM, Cote GM and Hornick JL. Contemporary sarcoma diagnosis, genetics and genomics. J Clin Oncol 36: 101-110, 2018.

12. Thampi S, Matthay KK, Goldsby R and DuBois SG: Adverse impact of regional lymph node involvement in osteosarcoma. Eur J Cancer 49: 3471-3476, 2013.

13. Lee JS, Fetsch JF, Wasdhal DA, Lee BP, Pritchard DJ and Nascimento AG: A review of 40 patients with extraskeletal osteosarcoma. Cancer 76: 2253-2259, 1995.

14. Ahmad SA, Patel SR, Ballo MT, Baker TP, Yasko AW, Wang X, Feig BW, Hunt KK, Lin PP, Weber KL, et al: Extraosseous osteosarcoma: Response to treatment and long term outcome. J Clin Oncol 20: 521-527, 2002.

15. Choi LE, Healey JH, Kuk D and Brennan MF: Analysis of outcomes in extraskeletal osteosarcoma: A review of fifty-three cases. J Bone Joint Surg Am 96: e2, 2014.

16. Longhi A, Bielack SS, Grimer R, Whelan J, Windhager R, Leithner A,Gronchi A, Biau D, Jutte P,Krieg AH, et al: Extraskeletal osteosarcoma: A European musculoskeletal oncology society study on 266 patients. Eur J Cancer 74: 9-16, 2017.

17. Paludo J, Fritchie K, Haddox CL, Rose PS, Arndt CAS, Marks RS, Galanis E, Okuno SH and Robinson SI: Extraskeletal Osteosarcoma: Outcomes and the Role of Chemotherapy. Am J Clin Oncol 41: 832-837, 2018

18. Wildgruber M, Sadick M, Müller-Wille R and Wohlgemuth WA: Vascular tumors in infants and adolescents. Insights Imaging 10: 30, 2019.

19. DeHart A and Richter G: Hemangioma: Recent advances. F1000Res 8: F1000 Faculty Rev-1926, 2019.

20. Walczak BE, Johnson CN and Howe BM: Myositis Ossificans. J Am Acad Orthop Surg 23: 612-622, 2015.

21. Sabatier R, Bouvier C, de Pinieux G, Sarran A, Brenot-Rossi I, Pedeutour F, Chetaille B, Viens P, Weiller PJ and Bertucci F: Low-grade extraskeletal osteosarcoma of the chest wall: Case report and review of literature. BMC Cancer 10: 645, 2010.

22. Yamashita K, Kohashi K, Yamada Y, Nishida Y, Urakawa H, Oda Y and Toyokuni S: Primary extraskeletal osteosarcoma: A clinicopathological study of 18 cases focusing on MDM2 amplification status. Hum Pathol 63: 63-69, 2017.

23. Makise N, Sekimizu M, Kubo T, Wakai S, Watanabe SI, Kato T, Kinoshita T,Hiraoka N, Fukayama M,Kawai A, et al: Extraskeletal osteosarcoma: MDM2 and H3K27me3 analysis of 19 cases suggest disease heterogeneity. Histopathology 73: 147-156, 2018.

24. Miwa S, Takeuchi A, Ikeda H, Shirai T, Yamamoto N, Nishida H, Hayashi K, Tanzawa Y, Kimura H, Igarashi K and Tsuchiya H: Prognostic value of histological response to chemotherapy in osteosarcoma patients receiving tumor-bearing frozen autograft. PLoS One 8: e71362, 2013. 